\title{
Prediction of Perceptual Quality for Mobile Video Using Fuzzy Inference Systems
}

\author{
Mohammed Alreshoodi, Student Member, IEEE, Emad Danish, Student Member, IEEE, John Woods, \\ Anil Fernando, Senior Member, IEEE, and Chamitha De Alwis
}

\begin{abstract}
Along with the rapid growth in consumer adoption of modern portable devices, video streaming is expected to dominate a large share of the global internet traffic in the near future. In the wireless communications domain, this trend creates considerable challenges to consumers' quality of experience (QoE). From a consumer-focused vision, predicting perceptual video quality is extremely important for QoE-based service provisioning. However, available QoE measurement techniques that adopt a full reference model are impractical in real-time transmission since they require the original video sequence to be available at the receiver's end. Therefore, the primary aim of this study is to present a cross-layer noreference prediction model for the perceptual quality of $3 D$ video in the wireless domain. The contributions of this study are twofold: first, the impact of selected quality of service (QoS) parameters from both encoding and network levels on $Q o E$ is investigated. Also, the obtained QoS/QoE correlation is backed by thorough statistical analysis. Second, a prediction model based on fuzzy logic inference systems (FIS) is developed by mapping chosen QoS parameters to the measured QoE. This model enables a non-intrusive prediction of $3 D$ visual quality. Conclusive results show a significantly high correlation between the predicted video quality and the objectively measured mean opinion scores (MOS). Objective MOS is also validated through methodical subjective assessments. For consumer's QoE, this study advances the development of reference-free video quality prediction models and QoE control methods for $3 D$ video streaming ${ }^{1}$.
\end{abstract}

Index Terms - QoE, QoS, consumer, estimation, H.264, MOS.

\section{INTRODUCTION}

Since modern portable consumer devices (tablets, smartphones, etc.) were brought into existence, user consumption of video content has been increasing. According to a recent research study [1], video is expected to dominate up to $90 \%$ of the global internet traffic by 2018 . This study also stated

\footnotetext{
${ }^{1}$ M. Alreshoodi is with the School of Computer Science and Electronic Engineering, University of Essex, Colchester, CO4 3SQ, UK (e-mail: mamalr@essex.ac.uk).

E. Danish is with the Department of Electronic Engineering, University of Surrey, Guildford, GU2 7XH, UK (e-mail: e.danish@surrey.ac.uk).

J. Woods is with the School of Computer Science and Electronic Engineering, University of Essex, Colchester, CO4 3SQ, UK (e-mail: woodjt@essex.ac.uk).

A. Fernando is with the Department of Electronic Engineering, University of Surrey, Guildford, GU2 7XH, UK (e-mail: w.fernando@surrey.ac.uk).

C. De Alwis is with the Faculty of Engineering, University of Sri Jayewardenepura, Nugegoda, Sri Lanka. (e-mail: c.dealwis@surrey.ac.uk).
}

that $61 \%$ of the global traffic will be wireless. In the future, this traffic will also contain $3 \mathrm{D}$ video which is even more resourceintensive [2]. Furthermore, this demand on wireless bandwidth is compounded by high expectations from the consumer with regard to quality of experience (QoE) [3], [4]. Therefore, QoE estimation, monitoring, and control are pressing requirements for wireless networks.

Perceptual video quality can be measured at the receiving terminal; however, this is impractical with full-reference (FR) QoE metrics since the reference video is absent at the receiving end. Therefore, it is essential to predict video quality in a noreference (NR) mode. NR models provide less accurate measurements than FR models, but NR measurements are sufficiently reliable for real-time video streaming.

Prediction of QoE is essential for consumer-centric service provisioning. It provides several advantages to both the consumer and the service provider. For example:

$\checkmark$ Through automated real-time QoE monitoring, service providers can control and maintain desired quality levels to the user through the management of controllable QoS parameters, such as video codec, bitrate, signal power, modulation, etc.

$\checkmark$ For service charging, QoE could be used as a criterion for quality-based billing that employs differentiated charging schemes in real-time.

$\checkmark$ More efficient QoE-based resource utilization can be achieved in terms of bandwidth utilization and power consumption [7].

QoE prediction, however, requires a firm understanding of those QoS factors that are the most influential on QoE [5], [6]. Hence, it is equally important to model the relation between QoS and QoE so that QoE can be predicted given QoS. There are several QoS factors that influence end-to-end video quality, but their joint effect is obscure and their interactions are believed to be nonlinear [3]. For this obscurity and nonlinearity, learning models represent a feasible approach to model the QoS/QoE correlation since they have the ability to learn then predict in a manner similar to human reasoning. Different learning-based techniques have been used by researchers to develop predictive QoE models. However, most of the research in this area have discussed partial solutions and have overlooked some influential QoS parameters across the video delivery layers.

In this paper, a cross-layer non-intrusive QoE prediction model is proposed. The model considers fuzzy logic inference 
systems (FIS) as a learning-based technique to estimate end-toend 3D video quality in the context of wireless video streaming. This paper extends the contributions in previous work [8] with three additional elements. Firstly, video spatial resolution has been added to the QoS parameters in the prediction model. Secondly, subjective assessments of the tested video sequences have been carried out to validate the obtained results from the objective metric used. Thirdly, statistical validation of the constructed datasets has been conducted using the analysis of variance technique (ANOVA).

The rest of this paper is organized as follows. A review of related research is presented section II. The experimental setup is described in section III. In section IV, QoE measurements collected from the simulations are presented, validated, and analyzed statistically. Section V is devoted to the methodology used in the proposed quality prediction model. In section VI the proposed prediction model is validated and performance evaluated. Finally, section VII concludes the paper.

\section{RELATED WORK}

Estimation of perceived quality of multimedia content in mobile environments is a significant issue for $\mathrm{CE}$ devices [5]; hence, it has been an area of significant interest to researchers in video quality. Within the scope of artificial intelligence (AI), learning-based techniques including various types of machine learning [9] have been the prime focus for developing objective QoE prediction models.

Some studies considered QoS parameters from the application layer only, such as video codec and bitrate [10]-[13]. Noreference (NR) algorithms were used to estimate peak-signal-tonoise-ratio (PSNR) [10], or to measure 2D quality using video quality assessment in the compressed domain (C-VQA) [11]. The reduced reference (RR) and full reference (FR) methods proposed, on the one hand they were based on parametric nonmachine learning algorithms [12], but on the other hand, they were based on machine learning [13].

Another group of research studies focused on QoS parameters solely from the network layer, such as packet loss and delay [9], [14], [15]. Again, either machine learning was used to assess QoS/QoE correlation [9], or a fuzzy expert system was used for QoE estimation [14], [15]. The majority employed the mean opinion score (MOS) as a quality measure.

However, for a broader prediction of video quality, hybrid models came to light and consolidated both application layer and network layer parameters [2]-[4], [16]-[18]. Khan et al. [3], [16] proposed a non-linear regression-based model to estimate video quality in PSNR normalized to MOS, and validated the model with subjective testing [3]. However, the study lacks the testing of spatial resolutions as a QoS factor. Fuzzy logic control was used in an application to an H.261 encoder to maximize QoE of video [19]. Another potential application of FIS is in QoE-based content-aware and energyefficient wireless resource allocation [20], [21]. A real-time estimator was proposed by Paudel et al. [17] utilizing random neural networks (RNN) as a prediction engine. Further contentfocused studies presented a RR metric for 3D video [2] based on PSNR, or just an investigation of QoS impact on QoE for videos encoded with high-efficiency-video-coding (HEVC) [4]. Joskowicz et al. [18] presented a mathematical parametric model for the prediction engine.

It is observed that existing proposals of video quality prediction tend to consider either the encoder's compression artifacts, or network impairments, or the features of video content, but rarely all three. Therefore, the proposed model in this paper extends existing work by addressing a group of QoS parameters not addressed so far in the context of $3 \mathrm{D}$ video.

\section{EXPERIMENTAL SETUP}

\section{A. Video Encoding}

Based on temporal activity, three classes of 3D video content are tested. The spatio-temporal classification metric in recommendation ITU-T P.910 [22] is used for this purpose. This technique extracts spatial and temporal features from a video sequence, and then assigns a spatial index (SI) and a temporal index (TI) based on the Sobel filter. The computed index indicates the spatial complexity and temporal activity of the video sequence. This technique is of low complexity, thus can classify videos in real-time. Consequently, three video sequences were chosen, one in each class, as listed in Table I. Each sequence is in YUV 4:2:0 format, of 25 (fps) and 200 frames in length, that is 8 seconds in time.

Both the color image and the depth map were encoded with the H.264/AVC video coding standard [23]. Configuration parameters of the encoder are depicted in Table II. Within the encoding process, network abstraction layer (NAL) units are encapsulated in real-time transport protocol (RTP) packets. It is also assumed that each RTP packet is encapsulated in one internet protocol (IP) packet on the network layer. Hence, packet loss rate (PLR) denotes the loss of video NAL units.

\section{B. QoS Parameters}

The chosen and simulated QoS parameters and their

TABLE I

VIDEO SEQUeNCES ChOSEN AND ClaSSES ASSIGNED

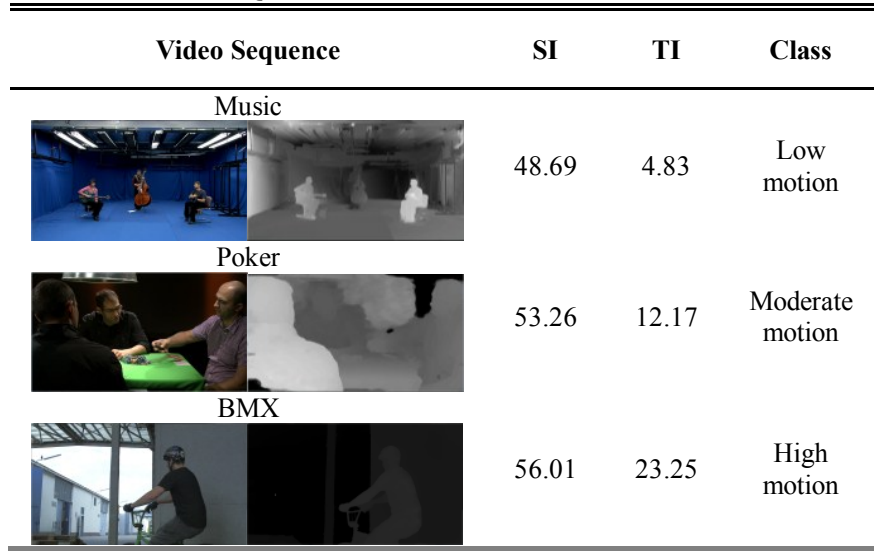


TABLE II

H.264/AVC VIDEO CODING PARAMETERS

\begin{tabular}{lc}
\hline \multicolumn{1}{c}{ Parameter } & Value \\
\hline Profile IDC & High (100) \\
Level IDC & $30(\mathrm{SD}), 32(\mathrm{qHD}, \mathrm{HD})$ \\
Sequence GoP & IPPP \\
Number of reference frames & 2 \\
Entropy coding & CAVLC \\
Search range & 32 \\
Slice mode & Packetized (bytes) \\
Output File Format & RTP packet \\
Rate control & Disabled \\
\hline
\end{tabular}

corresponding values are summarized in Table III. In addition to content type (CT), parameters from both the application layer and the physical layer were selected. Parameter values were designed carefully in order to generate a broad range of quality levels $(\mathrm{QoE})$ from poor to excellent to satisfy the diversity needed for the proposed prediction model. For increased data confidence, simulation of each tested condition (CT, R, QP, PLR, and MBL) is repeated 10 different times, such that the error trace starts at a displaced position of the coded bit-stream each time. This resembles real-life communications where errors could occur at any given point of transmission time. Also, this ensures that 2000 video frames are considered per each simulation condition. Consequently, once QoE of the 10 received videos is recorded, the mean $\mathrm{QoE}$ is calculated and a $95 \%$ confidence interval is established.

Considering the test conditions outlined in Table III which are repeated 10 times for 3 content types, a huge dataset of 10,800 tested conditions in total was constructed.

\section{Simulation Scene and the Wireless Error Model}

The objective is to model the effect of various QoS factors on perceived QoE across the media delivery chain. Hence, for endto-end 3D video quality estimation, QoS parameters from both the application and the physical layers are selected. This is because video quality is degraded by the distortions caused by both the video encoder and the access network.

Thus, to map the selected QoS parameters to corresponding QoE, the simulation scene was designed and conducted, as illustrated in Fig. 1. 3D video sequences in the form of 2D color image plus depth map are assigned a class identifier each according to their temporal activity. Later they are coded with H.264/AVC video compression standard [23], and then the coded video packets are simulated for wireless transmission

TABLE III

SIMULATED QOS PARAMETERS

\begin{tabular}{lc}
\hline \multicolumn{1}{c}{ Parameter } & Values \\
\hline Content type (CT) & Low, moderate, high motion \\
Spatial resolution (R) & SD $(720 \times 576)$, \\
& qHD $(960 \times 540)$, \\
Encoder quantization parameter (QP) & HD $(1280 \times 720)$ \\
Packet loss rate (PLR) & $16,24,32,40,48$ \\
& $0 \%, 0.1 \%, 1 \%, 2.5 \%, 5 \%$, \\
Mean burst length (MBL) & $7.5 \%, 10 \%$ \\
\hline
\end{tabular}

errors. The decoded video frames are then assessed for quality using a 3D video quality metric. Detailed descriptions of each stage are explained in the following subsections.

The wireless channel is simulated by introducing both random and burst packet losses to the transmitted packet stream, in order to analyze a broader range of simulation conditions. Random packet losses are uniformly distributed along the packet loss trace, while burst packet losses are distributed as bursts with a mean burst length (MBL), along the packet loss trace. Packet loss traces are generated based on the Gilbert-Elliot model [24] (a two-state Markov chain model) by varying the PLR and the MBL. In Table III, an MBL value of 1 depicts random packet losses, whereas other MBL values represent increasingly bursty conditions.

\section{QoE Measurement}

QoE is a broad concept that denotes users' levels of satisfaction. Of the various dimensions that constitute QoE, user's perception of the content consumed is considered the most influential dimension [6]. Hence, perceptual quality prediction of $3 \mathrm{D}$ video is the focus of this paper. For the sake of developing a concrete QoE prediction model, a large dataset and test conditions were constructed for this study. Therefore, an objective quality metric is used for quality assessment. However, to validate the quality measurements of this objective assessment, a subjective assessment was conducted as well using a selected subset of the test conditions.

\section{A. Objective Assessment}

Objective measurement is conducted using a validated FR perceptual 3D video quality metric [25]. This metric adopts the NTIA General Model [26] for the assessment of 2D color image. Known in the literature as "video quality metric" (VQM) [26], the NTIA General Model was independently evaluated by the video quality experts group (VQEG) and standardized by ANSI

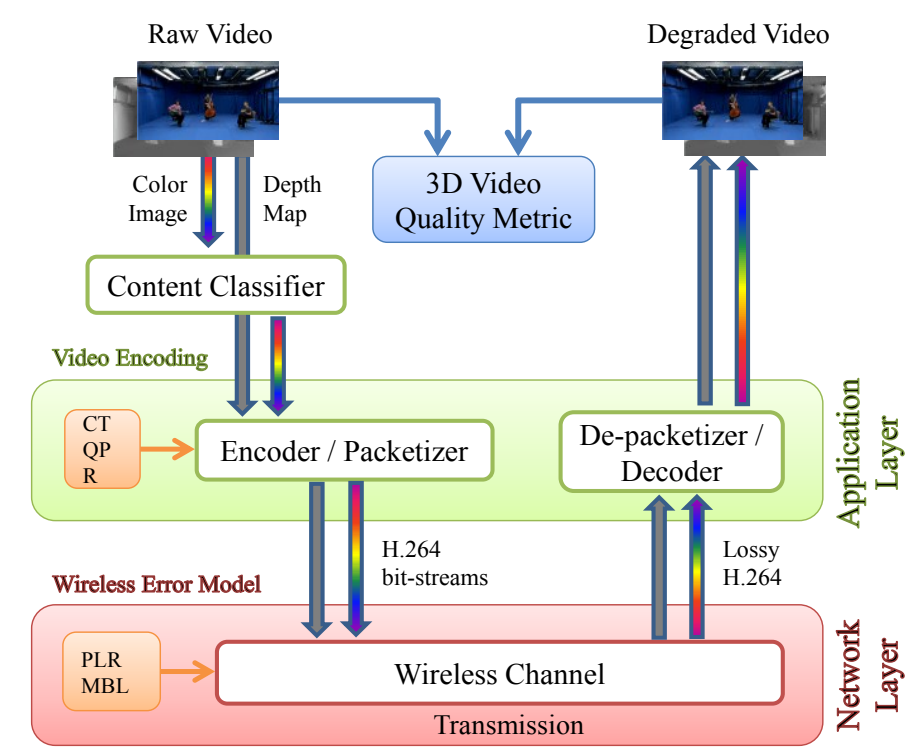

Fig. 1. Conceptual illustration of the simulation scene. 
and ITU. On the other hand, the depth map is assessed following the depth quality model [25], which measures the quality of depth signal based on the identification of dominant depth planes. The compound 3D quality is then determined through a joint mathematical model [25] using the measured VQM of 2D color image and the corresponding depth map. The quality scale on this metric is a continuous scale from 0 (complete loss) to 1 (original quality).

The use of this 3D quality metric, which adopts VQM within its engine for the $2 \mathrm{D}$ component, makes possible the use of several methods applicable to VQM analysis. For example, 2D quality measurements of the same video sequences are made available within the collected 3D dataset. Moreover, it makes possible the mapping of the 3D quality scale to subjective mean opinion score (MOS). Thus, to express the measured quality in corresponding subjective terms, measured quality values are normalized to MOS using [18]:

$M O S=5-4 V Q M$

or

$M O S=5-4(1-Q)$

Either (1) or (2) is used depending on the quality scale at hand. Equation (1) is used for VQM since on the VQM scale 1 is complete loss and 0 is original quality, whereas (2) is used for $Q$, the 3D quality measured, as the scale is opposite to that of VQM.

\section{B. Subjective Assessment}

Subjective testing is the most accurate method for measuring perceived video quality. However, the large number of test conditions required to formulate the proposed prediction model makes it extremely difficult to conduct subjective tests where video sequences need to be assessed by viewers. Therefore, the purpose of subjective assessment is to validate the obtained objective scores and assure their credibility, so they can be used confidently for the proposed QoE prediction model.

Subjective testing is performed based on the standard recommendation ITU-R BT.500-13 [27]. The subjective experiment was conducted using the $2 \mathrm{D}$ version of the test videos so they can be validated against the corresponding $2 \mathrm{D}$ results from objective scores. This validation can be drawn to 3D objective scores since the methods from ITU-R BT.500-13 [27] are also applicable in 3D scenarios [28].

Since the total test conditions for the dataset are 1080 conditions, a systematic approach was followed to select a subset designated for the subjective testing. The dataset used was constructed of objective measurements and the 2D HD video of each sequence was chosen to perform a balanced selection of conditions that spans the quality scale (0-to-1). The selection approach was based on the Kennard and Stone algorithm [29], which selects as the next sample the one that is most distant from those already selected samples. Thus, it covers the
TABLE IV

SUBJECTIVE MEAN OPINION SCORES [27]

\begin{tabular}{cccccc}
\hline \hline Quality & Bad & Poor & Fair & Good & Excellent \\
\hline MOS & 1 & 2 & 3 & 4 & 5 \\
\hline
\end{tabular}

experimental region uniformly and yields a flat distribution of the data. This guarantees that each value of each QoS parameter is covered among the whole sample space. 20 samples of each video content type plus the reference video were selected; hence the subjective dataset was formed of 63 sequences.

Proper consideration of relevant guidelines from ITU-R BT.500-13 [27] was taken throughout the subjective testing process. A panel of 21 expert and naïve viewers took the test of the single stimulus (SS) quality evaluation method, in a laboratory under a controlled and convenient environment. 2D HD videos were displayed on a 47" LED monitor, and the users marked their MOS responses on a continuous scale between 0 and 5, which was recorded in percentages from 0 to 100 . Later this continuous scale was normalized using (2) to the ITU-R BT.500-13 scale [27] illustrated in Table IV. At the beginning of the test session, the participants were trained with 5 selected video sequences, and then shown another 5 stabilizing sequences, both of which were discarded from the collected responses. Test sequences were randomly re-ordered to each user, and the reference video was hidden to them. The average time elapsed during test sessions was 17-19 minutes.

Mean opinions scores of the 21 observers with 95\% confidence intervals are shown in Fig. 2 for the 63 test conditions of HD videos.

\section{Correlation of Objective and Subjective Scores}

As a validation measure for the objective scores obtained through simulations, the correlation between subjective MOS and objective MOS for 2D videos is presented in Fig. 3. A Pearson correlation coefficient (PCC) of 0.92 indicates a high level of correlation and acknowledges the validity of the collected objective QoE data for 2D video.

Fig. 4 portrays a comparison of 21 test conditions for the "Poker" HD video with the scored MOS in each of the three datasets constructed in this paper. There is an apparent correlation between the three datasets.

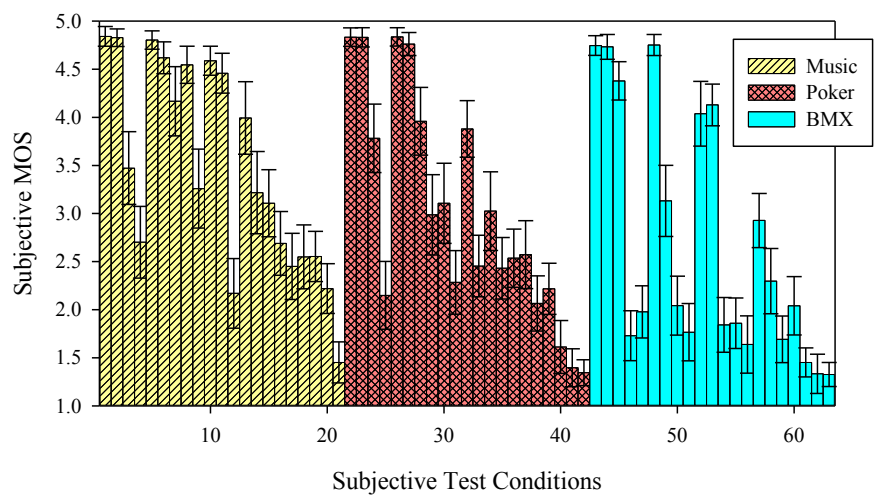

Fig. 2. Subjective MOS scores with $95 \%$ confidence intervals. 


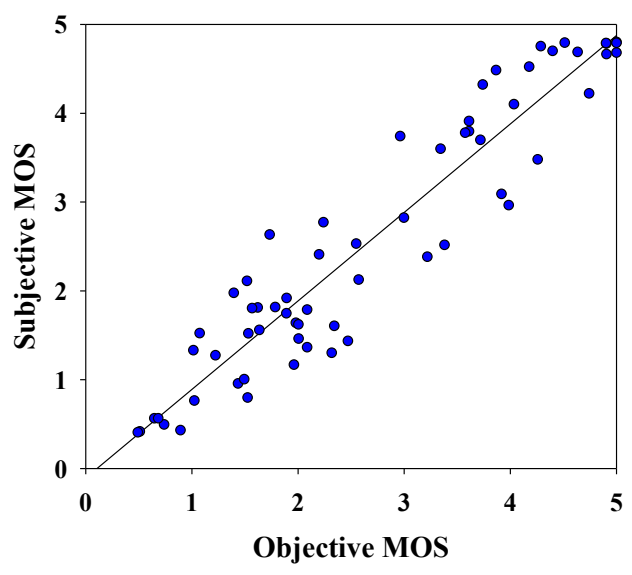

Fig. 3. Correlation of subjective MOS and objective MOS.

Furthermore, the PCC is computed for all 120 test conditions of "Poker" HD comparing the 2D objective and 3D objective data. The two datasets were highly correlated with a PCC of 0.9963. Consequently, it can be concluded that validation of the $2 \mathrm{D}$ objective dataset can be certainly drawn to the $3 \mathrm{D}$ objective dataset.

The 3D objective dataset is later used for learning and validation in the proposed FIS-based prediction system as explained in section V.

\section{D.Data Analysis with ANOVA}

The five QoS parameters identified in this paper are those described in Table III. To statistically establish the relationship between QoE and these five parameters, a 5-way analysis of variance (ANOVA) [30] test was carried out on the MOS dataset obtained by objective testing on 3D video. Hence, all the 1080 test conditions in the dataset were tested with 5-way repeated ANOVA. This is to determine the impact of all five parameters on MOS, as well as the interactions in between the parameters, i.e., their combined effect on MOS.

Table $\mathrm{V}$ shows the results obtained from the ANOVA analysis. A small p-value $(p \leq 0.01)$ indicates that MOS is significantly affected by the corresponding parameter [30]. This implies that all five parameters $(p$-value $=0$ ) have a significant effect on MOS. Furthermore, there are interaction effects between each pair of parameters, and each 2-way interaction is

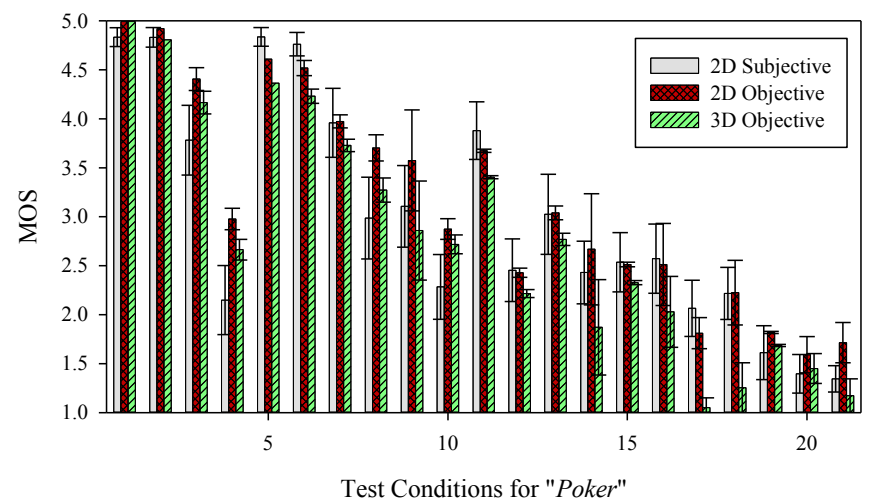

Fig. 4. Comparison of the three datasets for "Poker" HD video sequence. significant as well. With 3-way interactions, some parameters are of less impact $(p \geq 0.01)$ when combined. The 4-way interactions capture the 3 -way impact as well.

The most important parameter in the physical layer is PLR, which its impact on QoE is more than MBL. This is because the loss pattern of packet loss does, in fact, have a significant effect on the resulting distortion. In the case of random loss (PLR), frame dependency seems to play an influential role in propagating errors. However, for burst packet loss, the influence of frame dependency on error propagation decreases with the growth in average burst length.

The effect of varying MBL over different PLR is dependent on spatial resolution (R). Hence, for poor network conditions video resolution can be adapted to enhance a user's QoE. Moreover, in more bursty conditions adjusting QP with R would improve a user's perception decently. However, in such bursty conditions CT plays a considerable role as well.

Overall, ANOVA analysis showed that PLR is the most important parameter. The ANOVA results depicted in Table $\mathrm{V}$ confirmed there are interactions between the chosen five QoS parameters. This allowed the development of the quality prediction model by capturing the effects of QoS parameters.

\section{V.Quality Prediction Methodology}

In this paper, a no-reference QoE prediction model based on Fuzzy Logic Inference Systems (FIS) is proposed to estimate the impact of the encoding and network condition parameters on the video quality, i.e., the QoE. FIS is a well-known technique for

TABLE V ANOVA RESULTS FOR MAIN AND INTERACTION EFFECTS

\begin{tabular}{|c|c|c|c|c|}
\hline Source & $\begin{array}{l}\text { Sum of } \\
\text { squares }\end{array}$ & $\begin{array}{l}\text { Degree of } \\
\text { freedom }\end{array}$ & $\begin{array}{c}\text { F- } \\
\text { statistics }\end{array}$ & $\begin{array}{c}\text { p- } \\
\text { value }\end{array}$ \\
\hline $\mathrm{CT}$ & 0.9684 & 2 & 141.7391 & 0 \\
\hline $\mathrm{R}$ & 0.85 & 2 & 124.416 & 0 \\
\hline QP & 21.2487 & 4 & 1555.0708 & 0 \\
\hline PLR & 28.0041 & 5 & 1639.5704 & 0 \\
\hline MBL & 5.1769 & 3 & 505.1559 & 0 \\
\hline $\mathrm{CT} * \mathrm{R}$ & 0.5745 & 4 & 42.0471 & 0 \\
\hline $\mathrm{CT} * \mathrm{QP}$ & 0.6528 & 8 & 23.8862 & 0 \\
\hline CT*PLR & 0.3733 & 10 & 10.9266 & 0 \\
\hline $\mathrm{CT}^{*} \mathrm{MBL}$ & 0.0661 & 6 & 3.2242 & 0.0046 \\
\hline $\mathrm{R} * \mathrm{QP}$ & 0.2987 & 8 & 10.9295 & 0 \\
\hline $\mathrm{R} * \mathrm{PLR}$ & 0.2563 & 10 & 7.5038 & 0 \\
\hline $\mathrm{R} * \mathrm{MBL}$ & 0.1421 & 6 & 6.9318 & 0 \\
\hline QP*PLR & 8.609 & 20 & 126.0083 & 0 \\
\hline QP*MBL & 0.7432 & 12 & 18.1301 & 0 \\
\hline PLR*MBL & 1.8473 & 15 & 36.052 & 0 \\
\hline $\mathrm{CT} * \mathrm{R} * \mathrm{QP}$ & 0.2009 & 16 & 3.6755 & 0 \\
\hline CT*R*PLR & 0.1498 & 20 & 2.193 & 0.003 \\
\hline $\mathrm{CT} * \mathrm{R} * \mathrm{MBL}$ & 0.1667 & 12 & 4.0675 & 0 \\
\hline CT*QP*PLR & 0.2223 & 40 & 1.6265 & 0.0145 \\
\hline $\mathrm{CT} * \mathrm{QP} * \mathrm{MBL}$ & 0.1542 & 24 & 1.8808 & 0.0094 \\
\hline CT*PLR*MBL & 0.0907 & 30 & 0.8851 & 0.6429 \\
\hline $\mathrm{R} * \mathrm{QP} * \mathrm{PLR}$ & 0.1737 & 40 & 1.2715 & 0.1405 \\
\hline $\mathrm{R} * \mathrm{QP} * \mathrm{MBL}$ & 0.5197 & 24 & 6.3384 & 0 \\
\hline R*PLR*MBL & 0.6483 & 30 & 6.3258 & 0 \\
\hline QP*PLR*MBL & 0.4959 & 60 & 2.4194 & 0 \\
\hline $\mathrm{CT} * \mathrm{R} * \mathrm{QP} * \mathrm{PLR}$ & 0.192 & 80 & 0.7027 & 0.9671 \\
\hline $\mathrm{CT} * \mathrm{R} * \mathrm{QP} * \mathrm{MBL}$ & 0.3521 & 48 & 2.1474 & 0.0001 \\
\hline CT*R*PLR*MBL & 0.1903 & 60 & 0.9286 & 0.6249 \\
\hline $\mathrm{CT} * \mathrm{QP} * \mathrm{PLR} * \mathrm{MBL}$ & 0.3156 & 120 & 0.7699 & 0.9461 \\
\hline $\mathrm{R} * \mathrm{QP} * \mathrm{PLR} * \mathrm{MBL}$ & 0.83 & 120 & 2.0248 & 0 \\
\hline
\end{tabular}


user modeling that could imitate human reasoning using natural language in which words can imply ambiguous meanings [31]. It is considered as an extension to traditional set theory as statements could be partial truths, which means lying in between absolute truth and absolute falsity [32]. FIS includes four stages: fuzzifier, rule base, inference engine, and defuzzifier. FIS is powered with learned membership functions and a set of fuzzy inference rules. The rule base can be extracted from numerical data or predefined by experts. Upon rules' establishment, FIS maps the inputs to the outputs, and such mapping can be described numerically as $y=f(x)$ [32]. Fig. 5 shows a functional block of the proposed video quality prediction model.

The main objective of this approach is to design and implement a model that predicts the variation of the user satisfaction level as a function of the QoS parameters. FIS is computationally less intensive, a simple, transparent, and reasoning process. FIS outperforms other estimation techniques in terms of modeling capabilities and making decisions with imprecise information [33]. In addition, FIS provides a way of constructing controller algorithms by means of linguistic labels and linguistically interpretable rules in a user-friendly way closer to human thinking and perception. The methodology of designing the FIS-based model is explained in the following sub-sections.

\section{A. Identifying Inputs and Output}

In order to build learning sets for correlating QoS parameters with QoE, subjective and objective QoE tests were conducted as discussed in section IV. Five QoS parameters are chosen as inputs; PLR, MBL, CT, QP, and video resolution (R), while the output $(\mathrm{QoE})$ is a MOS score. The proposed methodology can also incorporate additional parameters. However, the interaction between these parameters is the determining factor for the quality of the delivered video and, consequently, the user satisfaction level.

\section{B. Design of Membership Functions}

Determining input/output membership functions is the first step of the fuzzy logic control process, where a fuzzy algorithm categorizes the information entering a system and assigns values that represent the degree of membership in those categories. The correlation between QoS parameters with the measured QoE is transferred into fuzzy membership functions and inference rules.

In this study, a membership function is derived using the

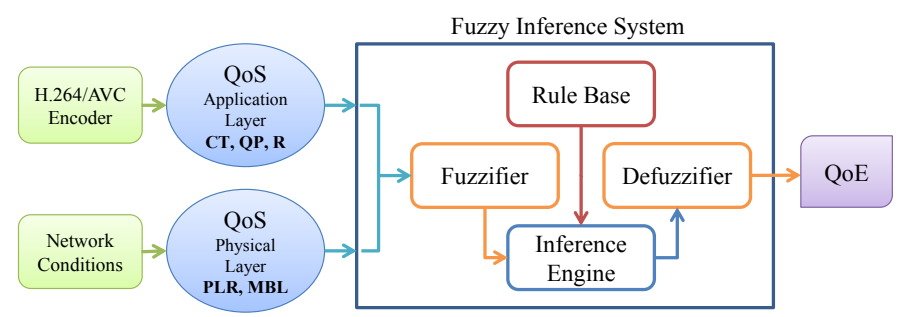

Fig. 5. Functional block diagram of the proposed FIS prediction model. probabilistic distribution function (PDF) [34]. Different PDFs are built for every QoS parameter. The probabilistic information was changed into a fuzzy set by dividing the PDF by its peak. The fuzzy set is expressed as a set of rules which take the form of linguistic expressions. Three fuzzy sets (low, moderate, high) were assigned to each of the fuzzy input variables. For the output, five fuzzy sets were assigned based on the MOS scores (bad, poor, fair, good and excellent). The fuzzy set is converted into an equivalent form (shape) of the membership function by using a curve fitting method [35]. The curve values of the membership functions represent the degree to which a particular QoS parameter value belongs to different MOS scores. The membership functions can take different forms; triangles, trapezoids, bell curves or any other shape as long as those shapes accurately represent the distribution of information. For this system, the triangular shape was chosen. The fuzzy set is converted into an equivalent triangular fuzzy set. Due to space limitations only two membership functions are illustrated in Fig. 6, (a) for PLR, (b) for MBL. In Fig. 7, the membership function of the output (QoE) is defined according to the standard MOS definition. Note that a membership value of 1 represents a high degree of membership to the corresponding class and a decreasing value represents deviation from the class.

\section{C.Fuzzy Rules Extraction}

In a FIS, a rule base is constructed to control the output variable. A fuzzy rule is a simple IF-THEN rule with a condition and a conclusion [32]. In this study, the used fuzzy inference system is Mamdani-type [36]. Fuzzy rules are derived by combining human knowledge and QoS parameters behavior with testing by a simulator. Based on the combinations of QoS metrics and their ratings, the impact of QoS variables on video quality (QoE) is estimated to one of the MOS scores (QoE). That is, an estimated QoE score is required to be associated with each combination of QoS parameter values. The following is a sample fuzzy rule for the proposed FIS:

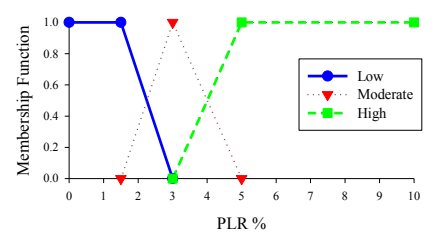

(a)

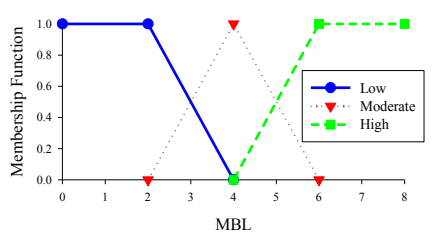

(b)
Fig. 6. Membership functions of (a) PLR and (b) MBL.

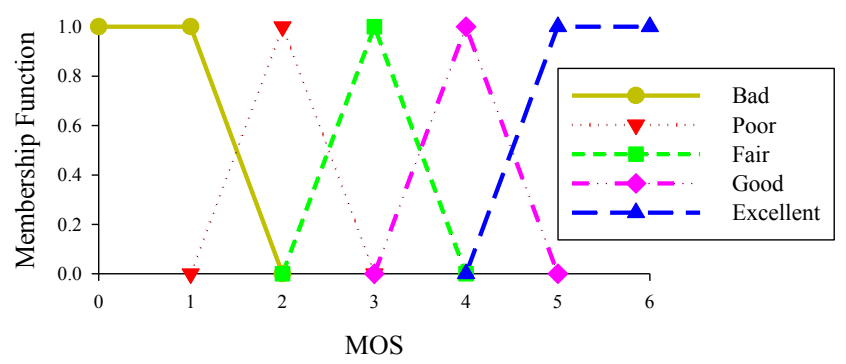

Fig. 7. Membership functions of the output (QoE). 
IF (CT is High motion) \& (MBL is High) \& (PLR is High) \& ( $Q P$ is Moderate) \& ( $R$ is Moderate) THEN ( $Q o E$ is Bad).

The fuzzy rules are generated by assigning weights to the QoS parameter values. For each combination, the rule weight is calculated as the sum of the weights of the QoS parameter values. The fuzzy rules and the combination of the results of the individual rules are evaluated by using fuzzy set operations, such as AND (intersection) and OR (union) [31]. The AND operator is used in this work, which is based on selecting the minimum value of the fuzzy sets.

After evaluating the result of each rule, these results are combined to obtain a final result. This step is called the inference engine. The results of individual rules are combined by the maximum algorithm [31]. This algorithm is the mostly used accumulation method that combines the results of individual rules by selecting the fuzzy set that achieves the greater membership value in the IF part of the rule.

The generation of the greatest possible number of rules is formed as $X^{n}$, where $X$ is the number of fuzzy sets and $n$ is the number of input variables. So, the maximum number of rules that can be extracted is $3^{5}$ possible rules. If a rule predicts more than one QoE class then the QoE class with the highest accuracy is considered to resolve the conflict between the rules.

\section{QoE Prediction}

After the inference step, the overall result is a fuzzy value.

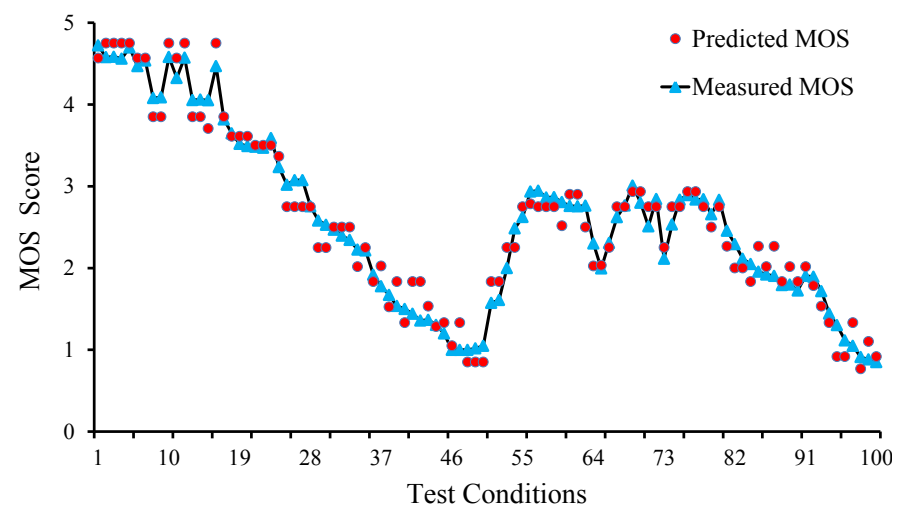

(a)

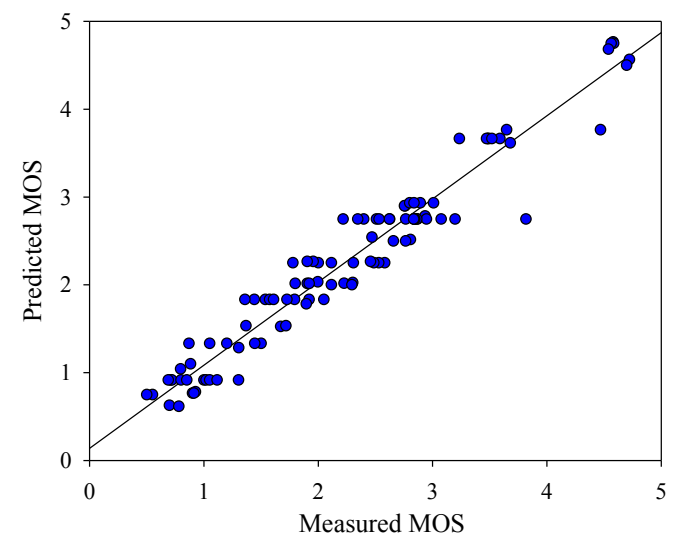

(b)

Fig. 8. Measured MOS vs. Predicted MOS.
This result should be defuzzified to obtain a final crisp output. The defuzzification is performed according to the membership function of the output. There are different defuzzification methods and the mostly used one is the center-of-gravity (COG). Mathematically, the COG can be expressed as:

$y=\frac{\sum_{i=1}^{M} S_{i} K_{i}}{\sum_{i=1}^{M} K_{i}}$

Where $y$ is the defuzzified output, $M$ is the number of rules, $S_{i}$ is the value of the output for rule $i, K_{i}$ is the inferred weight of the $i^{\text {th }}$ output membership function. In this work, a fuzzy logic toolbox was used to develop a simulation scenario with the designed membership functions and rules for validation using both the subjective and objective datasets.

\section{Validation and Performance Evaluation of the Proposed FIS-BASEd MODEL}

\section{A. Model Validation}

Once the relationships of individual QoS parameters and QoE are measured and recorded in the dataset, the $3 \mathrm{D}$ video dataset is used as inputs to the FIS-based model in order to map the QoS parameters to QoE scores. The proposed FIS-based model was validated using $R^{2}$ correlation and the root mean squared error (RMSE). The results obtained from the measured QoE (see section IV) were compared with the predicted QoE from FIS. Fig. 8 (a-b) shows the validation of the proposed system using line and scatter graphs. Each point in Fig. 8 (a) represents the predicted MOS of a particular 3D video clip and the line represents the measured QoE. $R^{2}$ scored 0.94 and RMSE was 0.109 . This indicates that predicted QoE is highly correlated with measured QoE. Thus, the proposed Fuzzy logic system significantly succeeds in predicting user's perception. These results show a consistent relationship between QoS and QoE for mobile video streaming.

\section{B. Model Performance Evaluation}

For evaluation of the FIS-based model's performance, two comparison tests were carried out. In the first test, the dataset constructed in section IV.D (of chosen parameters: PLR, MBL, and $\mathrm{QP}$ ) is used to compare the proposed FIS model with the random neural networks (RNN) technique [17], [37]. In the second test, an external dataset is used [16] as a further different comparison measure. In this test the proposed FIS model is compared against the nonlinear regression analysis (RA) model [16]. Results of both tests are shown in Table VI.

Overall, it can be noted that the proposed FIS-based prediction model outperformed the RNN-based and regressionbased models in terms of prediction accuracy. This is attributed to the precisely designed membership functions and inference rules. The more accurately defined the membership functions and inference rules the higher the prediction ability of the expert system [31]. Besides, the FIS takes advantage of being computationally less intensive, in addition to its transparent 
TABLE VI

Performance EVAluATION OF THE FIS-BASED MODEL

\begin{tabular}{lcc}
\hline \multicolumn{1}{c}{ Model } & RMSE & $\boldsymbol{R}^{2}$ \\
\hline FIS & 0.109 & 0.94 \\
RNN [17] & 0.218 & 0.91 \\
FIS (with external dataset) & 0.289 & 0.89 \\
RA [16] (with external dataset) & 0.355 & 0.87 \\
\hline
\end{tabular}

reasoning process.

Complexity analysis in terms of the time elapsed by the proposed FIS-based model, from the inputs to the output, is depicted in Table VII. The elapsed time was observed by running the model several times using different numbers of input samples. It is noted that the time elapsed is proportionate to the size of the input sample. The figures in Table VII were obtained by execution on a certain hardware configuration in which any change is likely to give different figures; however, the proportionate trend in these figures will still be preserved if a different hardware configuration is used.

\section{CONCLUSION AND FUTURE WORK}

In this paper, a zero-reference prediction model to estimate the quality of $3 \mathrm{D}$ video in wireless environments was developed. The model was developed using fuzzy logic inference systems (FIS). For end-to-end quality estimation, QoS parameters from both encoding and physical layers were identified. The QoS parameters were mapped to QoE scores by cross-layer simulation of the transmitted $3 \mathrm{D}$ video. The objectively measured QoE was validated through subjective assessments. Later, the QoS/QoE mapping dataset was statistically analyzed with 5-way ANOVA to confirm the impact of each chosen QoS parameter and to identify the most influential parameters. Finally, the dataset was fed into the FIS-based model for learning in preparation for prediction.

One conclusion to be drawn is that the choice of QoS parameters is crucial in achieving good prediction accuracy. For instance, PLR showed a more evident impact on quality as opposed to MBL. Also for video content type, PLR caused a greater impact on quality for higher motion content compared to lower motion.

A second substantial conclusion can be reached by validating the prediction model through the correlation of predicted QoE and measured QoE. The results showed a high prediction accuracy of the proposed FIS-based model in terms of $R^{2}$ correlation of 0.943 and RMSE of 0.10907 . This is of significance because the higher the accuracy in determining the expected level of QoE, the more proper decisions can be made regarding network resource provisioning whilst keeping the

TABLE VII

TIME ELAPSED FROM THE INPUTS TO THE OUTPUT

\begin{tabular}{ccccc}
\hline \hline Number of & \multicolumn{4}{c}{ Time elapsed (milliseconds) } \\
\cline { 2 - 5 } input samples & Maximum & Minimum & Average & Std. deviation \\
\hline 200 & 44 & 40 & 41 & 1.56 \\
600 & 63 & 58 & 60 & 1.44 \\
1000 & 98 & 92 & 96 & 2.05 \\
\hline
\end{tabular}

customer satisfied. The model was also evaluated against other comparable prediction models in which it showed better prediction accuracy.

This work complements the research efforts in QoE estimation with the objective to serve potential applications for QoE optimization and content provisioning to mobile users. Towards a more generic model, suggested future work includes additional QoS parameters to be investigated and perhaps incorporated. However, studying additional parameters should first investigate the extent to which each individual parameter would affect the user's perceived quality.

\section{REFERENCES}

[1] "Cisco Visual Networking Index: Forecast and Methodology, 2013-2018," Cisco, USA, White Paper FLGD 11684, Jun. 2014.

[2] C. T. E. R. Hewage and M. G. Martini, "Reduced-reference quality assessment for 3D video compression and transmission," IEEE Trans. Consumer Electron., vol. 57, no. 3, pp. 1185-1193, Aug. 2011.

[3] A. Khan, L. Sun, and E. Ifeachor, "QoE prediction model and its application in video quality adaptation over UMTS networks," IEEE Trans. Multimed., vol. 14, no. 2, pp. 431-442, Apr. 2012.

[4] J. Nightingale, Q. Wang, C. Grecos, and S. Goma, "The impact of network impairment on quality of experience (QoE) in H.265/HEVC video streaming," IEEE Trans. Consumer Electron., vol. 60, no. 2, pp. 242-250, May 2014.

[5] U. Reiter, "Perceived quality in consumer electronics - from quality of service to quality of experience," in Proc. IEEE International Symposium on Consumer Electronics, Kyoto, Japan, pp. 958-961, May 2009.

[6] "Triple-play services quality of experience (QoE) requirements," DSL Forum, Technical Report TR-126, Dec. 2006.

[7] E. Danish, A. Fernando, O. Abdul-Hameed, M. Alshamrani, and A. Kondoz, "Perceptual QoE based resource allocation for mobile 3D video communications," in Proc. IEEE International Conference on Consumer Electronics, Las Vegas, USA, pp. 454-455, Jan. 2014.

[8] M. Alreshoodi, E. Danish, J. Woods, A. Fernando, and C. de Alwis, "Prediction of perceptual quality for mobile 3D video using fuzzy inference systems," in Proc. IEEE International Conference on Consumer Electronics, Las Vegas, USA, pp. 141-142, Jan. 2015.

[9] M. S. Mushtaq, B. Augustin, and A. Mellouk, "Empirical study based on machine learning approach to assess the QoS/QoE correlation," in Proc. European Conference on Networks and Optical Communications, Vilanova i la Geltru, pp. 1-7, Jun. 2012.

[10] A. Eden, "No-reference estimation of the coding PSNR for H.264-coded sequences," IEEE Trans. Consumer Electron., vol. 53, no. 2, pp. 667-674, May 2007.

[11] X. Lin, H. Ma, L. Luo, and Y. Chen, "No-reference video quality assessment in the compressed domain," IEEE Trans. Consumer Electron., vol. 58, no. 2, pp. 505-512, May 2012.

[12] S.-O. Lee, K.-S. Jung, and D.-G. Sim, "Real-time objective quality assessment based on coding parameters extracted from H.264/AVC bitstream," IEEE Trans. Consumer Electron., vol. 56, no. 2, pp. 1071-1078, May 2010.

[13] H. Malekmohamadi, W. A. C. Fernando, E. Danish, and A. M. Kondoz, "Subjective quality estimation based on neural networks for stereoscopic videos," in Proc. IEEE International Conference on Consumer Electronics, Las Vegas, USA, pp. 107-108, Jan. 2014.

[14] J. Pokhrel, B. Wehbi, A. Morais, A. Cavalli, and E. Allilaire, "Estimation of QoE of video traffic using a fuzzy expert system," in Proc. IEEE Consumer Communications and Networking Conference, Las Vegas, USA, pp. 224229, Jan. 2013.

[15] M. Alreshoodi and J. Woods, "An empirical study based on a fuzzy logic system to assess the QoS/QoE correlation for layered video streaming," in Proc. IEEE International Conference on Computational Intelligence and Virtual Environments for Measurement Systems and Applications, Milan, Italy, pp. 180-184, Jul. 2013.

[16] A. Khan, L. Sun, E. Ifeachor, J. O. Fajardo, and F. Liberal, "Video quality prediction model for H.264 video over UMTS networks and their application in mobile video streaming," in Proc. IEEE International Conference on Communications, Cape Town, South Africa, pp. 1-5, May 2010. 
[17] I. Paudel, J. Pokhrel, B. Wehbi, A. Cavalli, and B. Jouaber, "Estimation of video QoE from MAC parameters in wireless network: a random neural network approach," in Proc. International Symposium on Communications and Information Technologies, Incheon, pp. 51-55, Sep. 2014.

[18] J. Joskowicz, R. Sotelo, and J. C. L. Ardao, "Towards a general parametric model for perceptual video quality estimation," IEEE Trans. Broadcast., vol. 59, no. 4, pp. 569-579, Dec. 2013.

[19] A. Bellini, A. Leone, R. Rovatti, E. Franchi, and N. Manaresi, "Analog fuzzy implementation of a perceptual classifier for videophone sequences," IEEE Trans. Consumer Electron., vol. 42, no. 3, pp. 787-794, Aug. 1996.

[20] E. Danish, V. De Silva, A. Fernando, C. de Alwis, and A. Kondoz, "Content aware resource allocation in OFDM systems for energy efficient video transmission," in Proc. IEEE International Conference on Consumer Electronics, Las Vegas, USA, pp. 456-457, Jan. 2014.

[21] E. Danish, V. Silva, A. Fernando, C. Alwis, and A. Kondoz, "Content-aware resource allocation in OFDM systems for energy-efficient video transmission," IEEE Trans. Consumer Electron., vol. 60, no. 3, pp. 320-328, Aug. 2014.

[22] "Subjective video quality assessment methods for multimedia applications," ITU, Switzerland, Recommendation ITU-T P.910, Apr. 2008.

[23] "Advanced video coding for generic audiovisual services," ITU, Switzerland, Recommendation ITU-T H.264, Feb. 2014

[24] E. O. Elliott, "Estimates of error rates for codes on burst-noise channels," Bell Syst. Tech. J., vol. 42, no. 5, pp. 1977-1997, Sep. 1963.

[25] S. L. P. Yasakethu, S. T. Worrall, D. V. S. X. De Silva, W. A. C. Fernando, and A. M. Kondoz, "A compound depth and image quality metric for measuring the effects of packet loss on 3D video," in Proc. IEEE International Conference on Digital Signal Processing, Corfu, Greece, pp. 1-7, Jul. 2011.

[26] M. H. Pinson and S. Wolf, "A new standardized method for objectively measuring video quality," IEEE Trans. Broadcast., vol. 50, no. 3, pp. 312322, Sep. 2004.

[27] "Methodology for the subjective assessment of the quality of television pictures," ITU, Switzerland, Recommendation ITU-R BT .500-13, Jan. 2012.

[28] "Subjective assessment of stereoscopic television pictures," ITU, Switzerland, Recommendation ITU-R BT.1438, Mar. 2000.

[29] R. W. Kennard and L. A. Stone, "Computer aided design of experiments," Technometrics, vol. 11, no. 1, pp. 137-148, 1969.

[30] G. W. Snecdecor and W. G. Cochran, Statistical Methods, 8th ed., Wiley, 1991.

[31] J. Bih, "Paradigm shift - an introduction to fuzzy logic," IEEE Potentials, vol. 25, no. 1, pp. 6-21, Jan. 2006.

[32] J. M. Mendel, "Fuzzy logic systems for engineering: a tutorial," Proc. IEEE, vol. 83, no. 3, pp. 345-377, Mar. 1995.

[33] A. R. Gray and S. G. MacDonell, "A comparison of techniques for developing predictive models of software metrics," Inf. Softw. Technol., vol. 39, no. 6, pp. 425-437, 1997.

[34] B. S. L. P. de Lima and N. F. F. Ebecken, "A comparison of models for uncertainty analysis by the finite element method," Finite Elem. Anal. Des., vol. 34, no. 2, pp. 211-232, 2000.

[35] M. B. Anoop, K. B. Rao, and S. Gopalakrishnan, "Conversion of probabilistic information into fuzzy sets for engineering decision analysis," Comput. Struct., vol. 84, no. 3-4, pp. 141-155, 2006.

[36] E. H. Mamdani and S. Assilian, "An experiment in linguistic synthesis with a fuzzy logic controller," Int. J. Man-Mach. Stud., vol. 7, no. 1, pp. 1-13, 1975.

[37] E. Gelenbe, "Random neural networks with negative and positive signals and product form solution," Neural Comput., vol. 1, no. 4, pp. 502-510, Dec. 1989.

\section{BIOGRAPHIES}

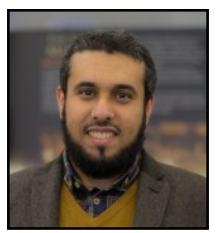

Mohammed Alreshoodi (S'13) received the B.Sc. Computer Science degree in 2005 from Qassim University, Buraydah, Saudi Arabia. The M.Sc. (Hons.) Computer Networking and Information in 2011, from the University of Essex. Currently, he is a Ph.D. researcher in Computer Science and Electronic Engineering School, University of Essex, Colchester, UK. His research interests are; computer networking, telecommunication, quality of experience and quality of service in the field of multimedia streaming.

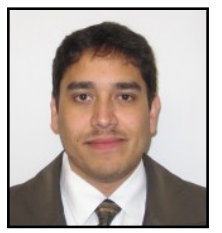

Emad A. Danish (S'13) received the B.Sc. Engineering degree in 1997, the M.Sc. (Hons.) Engineering degree in 2004, in Electrical and Computer Engineering from King Abdulaziz University, Jeddah, Saudi Arabia. Currently, he is a Ph.D. researcher in the Centre for Vision, Speech and Signal Processing, University of Surrey, Guildford, UK. His research interests include consumer's perception driven multimedia communications, with main focus on mobile video transmission, energy and bandwidth efficiency, QoE and Quality-of-Business.

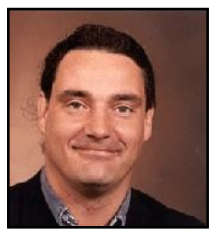

John C. Woods received the B.Eng. (Hons.) degree (first class) in 1996 and the Ph.D. degree in 1999 from the University of Essex, Colchester, UK. He has been a Lecturer in the Department of Computer Science and Electronic Systems Engineering, University of Essex, since 1999. Although his field of expertise is image processing, he has a wide range of interests including telecommunications, autonomous vehicles, and robotics. He is currently investigating local positioning systems and was recently a co-signatory to a European grant examining segmentation and tracking. He is a founding member of the Gridswarm consortium at Essex. He is a Consultant to the Ngee Ann Polytechnic in Singapore for augmented MPEG-4 over digital TV \& flight systems. Dr. Woods is a member of the IET.

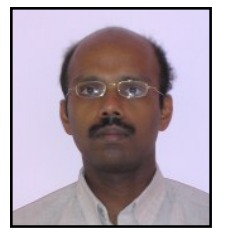

Anil Fernando (S'98-M'01-SM'03) received the B.Sc. Engineering degree (First class) in Electronic and Telecommunications Engineering from the University of Moratuwa, Sri Lanka in 1995 and the MEng degree (Distinction) in Telecommunications from Asian Institute of Technology (AIT), Bangkok, Thailand in 1997. He completed his $\mathrm{PhD}$ at the Department of Electrical and Electronic Engineering, University of Bristol, UK in February 2001. Currently, he is a reader in signal processing at the University of Surrey, UK. His current research interests include video communications, cloud communications, video coding, Quality of Experience (QoE), intelligent video encoding for wireless communications, resource allocations, channel coding and modulation schemes for wireless channels. He is a senior member of IEEE and a fellow of the HEA, UK and a member of the EPSRC College.

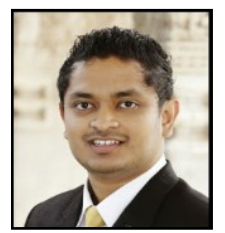

Chamitha De Alwis received the B.Sc. Engineering degree (First class) in Electronic and Telecomms. Engineering from the University of Moratuwa, Sri Lanka in 2009. He was awarded a Ph.D. By the Centre for Vision, Speech and Signal Processing, University of Surrey, Guildford, UK in 2014. Presently he is a senior lecturer in the University of Sri Jayewardenepura, Sri Lanka. His research interests include network coding, multimedia and wireless communication. 